\title{
Correction to: On minimum sum representations for weighted voting games
}

\author{
Sascha Kurz ${ }^{1}$
}

Published online: 31 May 2018

(C) Springer Science+Business Media, LLC, part of Springer Nature 2018

\section{Correction to: Ann Oper Res (2012) 196:361-369 https://doi.org/10.1007/s10479-012-1108-3}

\section{Introduction}

A weighted voting game is a yes-no voting system specified by nonnegative voting weights $w_{i} \in \mathbb{R}_{\geq 0}$ for the voters and a quota $q \in \mathbb{R}_{>0}$. A proposal is accepted iff $w(Y):=\sum_{i \in Y} w_{i} \geq$ $q$, where $Y$ is the set of voters which are in favor of the proposal. Restricting weights and quota to integers poses the question for minimum sum representations, where the sum of weights of all voters is minimized. For at most 7 voters, these representations are unique. For 8 voters, there are exactly 154 weighted voting games with two minimum sum integer weight representations. Two voters $i$ and $j$ are called equivalent if for all subsets $S \subseteq$ $\{1, \ldots, n\} \backslash\{i, j\}$ we have that $w(S \cup\{i\})$ and $w(S \cup\{j\})$ either both are strictly less than $q$ or both values are at least $q$. Adding the extra condition that equivalent voters should get the same weight, we speak of minimum sum representations preserving types. For $n=8$ voters, those representations are always unique, which is different for $n \geq 9$ voters. In Kurz (2012), we have determined the number of different weighted voting games with $n=9$ voters and then extracted those that do not admit a unique minimum sum representation. In our implementation of the proposed enumeration algorithm, we unfortunately relied on read and write accesses to a hard disk without further checking the validity of these operations. From the independent determination of the number of weighted games with $n=9$ voters in Kartak et al. (2015), we have learned that some weighted games were missed during the enumeration process. We have carefully traced back the differences and pinned down the technical reason for the erroneous. The algorithmic approach is not affected. Rerunning the

The original article can be found online at https://doi.org/10.1007/s10479-012-1108-3.

Sascha Kurz

sascha.kurz@uni-bayreuth.de

1 Department of Mathematics, Physics, and Computer Science, University of Bayreuth, 95440 Bayreuth, Germany 
algorithm, checking hard disk operations, we are able to verify the number of 993,061,482 weighted voting games. In the next section, we report the corrected statistics with respect to minimum sum representations.

\section{Corrected results}

Instead of 989,913,344 there are 993,061,482 weighted voting games for $n=9$ voters so that Tables 3, 4 and 5 from Kurz (2012) need to be corrected. The data of Table 6 remain valid. The corrected tables are given as follows (Tables 1,2,3).

In some cases, the power distribution of a weighted game can be completed to a representation by choosing a suitable quota. As additional information, we have enumerated the number of those cases for the Shapley-Shubik SSI, Penrose-Banzhaf BZI, Johnston Jo,

Table 1 Number of weighted voting games for 9 voters without a unique minimum sum representation by type and number of representations

\begin{tabular}{lrrr}
\hline Type & \multicolumn{1}{c}{3} & \multicolumn{1}{c}{$\sum$} \\
\hline 1 & 62,432 & 624 & 63,056 \\
2 & 0 & 492 & 492 \\
3 & 12,838 & 0 & 12,838 \\
4 & 0 & 200 & 200 \\
$\sum$ & 75,270 & 1316 & 76,586 \\
\hline
\end{tabular}

Table 2 Number of weighted voting games for 9 voters without a unique minimum sum representation by the number of representations and equivalence classes of voters

\begin{tabular}{lrrrrrr}
\hline Equivalence classes & \multicolumn{1}{l}{8} & \multicolumn{1}{c}{8} & \multicolumn{1}{c}{6} & \multicolumn{1}{c}{6} & \multicolumn{1}{c}{4} \\
\hline 2 representations & 5718 & 35,864 & 24,715 & 7659 & 1234 & 80 \\
3 representations & 0 & 402 & 500 & 330 & 76 & 8 \\
\hline
\end{tabular}

Table 3 Number of weighted voting games for 9 voters without a unique minimum sum representation preserving types by the number of equivalence classes of voters

\begin{tabular}{lllllll}
\hline Equivalence classes & 9 & 8 & 7 & 6 & 5 & $\sum$ \\
\hline 2 representations & 5718 & 4992 & 2134 & 392 & 14 & 13,250 \\
\hline
\end{tabular}

Table 4 Number of different power vectors of weighted voting games

\begin{tabular}{lllllrrrrr}
\hline$n$ & 1 & 2 & 3 & \multicolumn{1}{l}{4} & \multicolumn{1}{c}{5} & \multicolumn{1}{c}{6} & \multicolumn{1}{c}{7} & \multicolumn{1}{c}{8} & \multicolumn{1}{c}{9} \\
\hline SSI & 1 & 3 & 8 & 25 & 104 & 733 & 7780 & 113,804 & $2,445,026$ \\
BZI & 1 & 3 & 8 & 25 & 108 & 841 & 13,570 & 404,047 & $20,625,696$ \\
Jo & 1 & 3 & 8 & 22 & 74 & 289 & 1328 & 6927 & 42,187 \\
PGI & 1 & 3 & 8 & 22 & 71 & 213 & 788 & 4413 & 49,437 \\
DP & 1 & 3 & 8 & 25 & 87 & 278 & 1019 & 5695 & 64,002 \\
all & 1 & 3 & 8 & 25 & 117 & 1111 & 29,373 & $2,730,164$ & $993,061,482$ \\
\hline
\end{tabular}


Public Good PGI, and Deegan-Packel index DP in Table 4. In the line named "all", we have stated to total number of weighted games, i.e., the fraction of games permitting such a representation drastically decreases.

\section{References}

Kartak, V. M., Ripatti, A. V., Scheithauer, G., \& Kurz, S. (2015). Minimal proper non-IRUP instances of the one-dimensional cutting stock problem. Discrete Applied Mathematics, 187, 120-129.

Kurz, S. (2012). On minimum sum representations for weighted voting games. Annals of Operations Research, 196(1), 361-369. 